\title{
The acute effects of high-fat meals enriched with EPA or DHA $v$. oleic acid on cardiac output and other haemodynamics at rest and during exercise in healthy young men
}

\author{
V. G. Rontoyanni, S. Pombo-Rodrigues, A. Appleton, R. Chung, W. L. Hall and T. A. B. Sanders \\ Nutritional Sciences Division, King's College London, 150 Stamford Street, London SE1 9NH, UK
}

Recent research conducted by our group has suggested an acute improvement in the vascular tone of large arteries by adding EPA (20: $5 n-3)$ to a high-fat meal ${ }^{(1)}$. The acute effects of a single dose of EPA and/or DHA (22: $6 n$-3) on cardiac output (CO) are uncertain.

The effects on $\mathrm{CO}$ of high-fat meals ( $50 \mathrm{~g}$ fat) containing high-oleic sunflower oil enriched with $5 \mathrm{~g}$ either EPA or DHA compared with high-oleic sunflower oil only (control) at rest and during exercise are reported. Healthy non-smoking 18-45-year-old males ( $n$ 22) were randomised to each treatment, with at least 1 week between treatments. $\mathrm{CO}$ and blood pressure (BP) were measured at 0 (fasting), $1,2,3$ and $5 \mathrm{~h}$ following each test meal and during $12 \mathrm{~min}$ of multi-stage bicycle ergometry $(25 \mathrm{~W}, 50 \mathrm{~W}, 75 \mathrm{~W}, 100 \mathrm{~W}$, each of $3 \mathrm{~min}$ duration) at $5 \mathrm{~h}$.

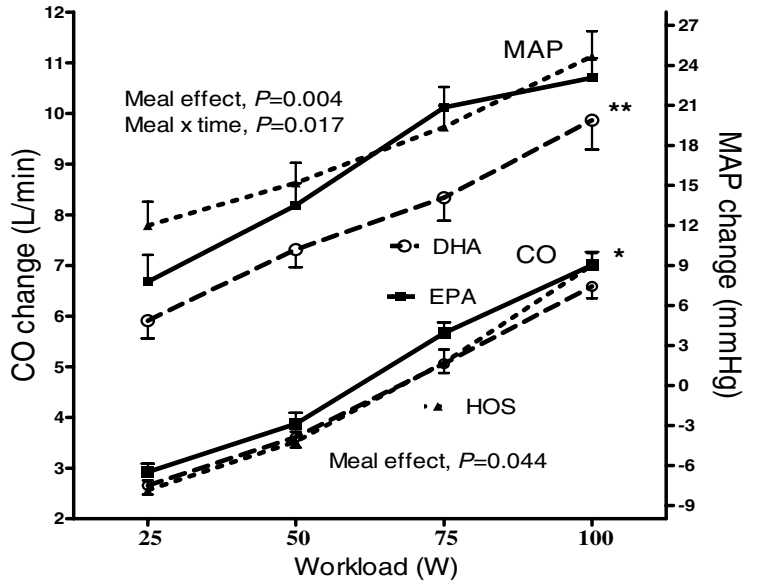

Fig. 1. Changes in exercise cardiac output $(\mathrm{CO}) \&$ mean arterial pressure (MAP) from fasting $(0 \mathrm{~h})$ after EPA, DHA and control meals in healthy men. Repeated measures ANOVA of the mean changes from $0 \mathrm{~h}$. Values are means with SEM. $*$ EPA vs DHA, $P<0.05 ; *$ DHA vs Control, $P=0.001$

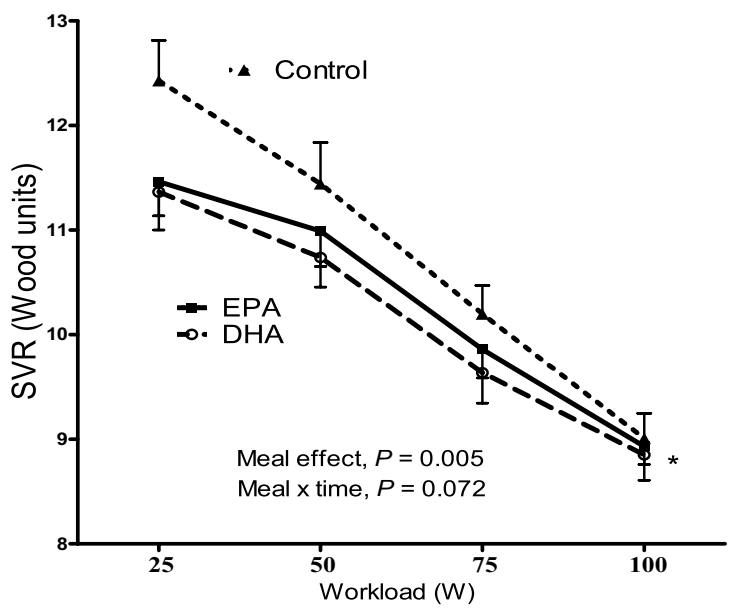

Fig. 2. Systemic vascular resistance (SVR) responses during exercise after EPA, DHA and control meals in healthy men. Repeated measures ANOVA of the means. Values are means with SEM. *DHA vs Control, $P<0.01$

Resting CO significantly increased from 5.6 ( $\mathrm{SE} 0.17$ ) $1 / \mathrm{min}$ at $0 \mathrm{~h}$ by 0.42 (95\% CI $0.15,0.69$ ) $1 /$ min $5 \mathrm{~h}$ postprandially (time effect, $P<0.05)$ on all meal treatments.

DHA resulted in smaller increases in exercise systolic, diastolic BP and mean arterial pressure (Fig. 1) compared with control as a result of a greater decrease in systemic vascular resistance (SVR; Fig. 2); the difference between DHA and control was 5.7 (95\% CI 0.9 , $10.5) \mathrm{mmHg}$ in systolic BP, $5.2(95 \%$ CI 2.5, 7.9) $\mathrm{mmHg}$ in diastolic BP and $5.8(95 \%$ CI 1.7, 9.9) \% in SVR. EPA induced a greater increase in exercise CO (Fig. 1) compared with DHA by 0.39 (95\% CI $0.03,0.74) 1 / \mathrm{min}$, as a result of an increase in stroke volume. Since EPA tended, although not significantly, to cause a greater increase by 0.33 (95\% CI $-0.16,0.82) 1 / \mathrm{min}$ in exercise CO and a greater fall by 4.3 (95\% CI - 0.7, 9.2) \% in exercise SVR compared with control, exercise BP responses did not differ between EPA and control.

The present study indicates that EPA and DHA may influence cardiovascular reactivity to dynamic exercise in different ways. Mainly DHA and possibly EPA appear to promote exercise vasodilatation with DHA imposing a smaller myocardial workload than EPA during exercise. 